\title{
A Note on Generalized Sasakian Space Forms with Interpolating Sesqui-Harmonic Legendre Curves
}

\author{
Fatma Karaca*
}

\begin{abstract}
We classify the curvature of interpolating sesqui-harmonic Legendre curves in generalized Sasakian space forms. We investigate the necessary and sufficient conditions for these types of curves in nine cases to be interpolating sesqui-harmonic.

Keywords: Generalized Sasakian space form; Legendre curve; interpolating sesqui-harmonic curve.

AMS Subject Classification (2020): Primary: 53C25 ; Secondary: 53C40.

${ }^{*}$ Corresponding author
\end{abstract}

\section{Introduction}

Biharmonic maps $\varphi:(M, g) \rightarrow(N, h)$ between Riemannian manifolds are well known a natural generalization of the harmonic maps [7]. Biharmonic maps are a critical point of the bienergy functional

$$
E_{2}(\varphi)=\int_{\Omega}\|\tau(\varphi)\|^{2} d \nu_{g}
$$

where

$$
\tau(\varphi)=\operatorname{tr}(\nabla d \varphi)
$$

is called the tension field of $\varphi$ [7]. The Euler-Lagrange equation for $E_{2}(\varphi)$ is

$$
\tau_{2}(\varphi)=\operatorname{tr}\left(\nabla^{\varphi} \nabla^{\varphi}-\nabla_{\nabla}^{\varphi}\right) \tau(\varphi)-\operatorname{tr}\left(R^{N}(d \varphi, \tau(\varphi)) d \varphi\right),
$$

which is the bitension field of $\varphi$ [8]. The equation $\tau_{2}(\varphi)=0$ is called biharmonic equation.

Interpolating sesqui-harmonic maps $\varphi:(M, g) \rightarrow(N, h)$ between Riemannian manifolds are defined that interpolated between the actions for harmonic and biharmonic maps [6]. The map $\varphi$ is called interpolating sesquiharmonic if it is a critical point of $E_{\delta_{1}, \delta_{2}}(\varphi)$

$$
E_{\delta_{1}, \delta_{2}}(\varphi)=\delta_{1} \int_{\Omega}\|d \varphi\|^{2} d \nu_{g}+\delta_{2} \int_{\Omega}\|\tau(\varphi)\|^{2} d \nu_{g}
$$

where $\delta_{1}, \delta_{2} \in \mathbb{R}$ [6]. The interpolating sesqui-harmonic map equation is given by

$$
\tau_{\delta_{1}, \delta_{2}}(\varphi)=\delta_{2} \tau_{2}(\varphi)-\delta_{1} \tau(\varphi)=0
$$

for $\delta_{1}, \delta_{2} \in \mathbb{R}$ [6]. If variations of the equation (1.3) that are normal to the image $\varphi(M) \subset N$ and $\delta_{2}=1, \delta_{1}>0$ then, an interpolating sesqui-harmonic map turns to biminimal [12].

In [6], Branding introduced an action functional for maps between Riemannian manifolds that interpolate between the actions for harmonic and biharmonic maps and studied interpolating sesqui-harmonic curves in a

Received : 18-11-2019, Accepted : 14-02-2020 
3-dimensional sphere. In [5], the same author studied a conservation law and used it to show the smoothness of weak solutions for a spherical target and found several classification results for interpolating sesqui-harmonic maps. In [10], the author, Özgür and De studied interpolating sesqui-harmonic Legendre curves in Sasakian space forms. In [16], Özgür and Güvenç studied biharmonic Legendre curves in generalized Sasakian space forms. Motivated by the above studies, in the present paper, we investigate interpolating sesqui-harmonic Legendre curves in generalized Sasakian space forms. We find the necessary and sufficient conditions for these types of curves in nine cases to be interpolating sesqui-harmonic.

\section{Preliminaries}

Let $N^{2 n+1}=\left(N^{2 n+1}, \phi, \xi, \eta, g\right)$ be an almost contact metric manifold with almost contact metric structure $(\phi, \xi, \eta, g)$. A manifold $\left(N^{2 n+1}, \phi, \xi, \eta, g\right)$ is called a Sasakian manifold if it is normal, that is,

$$
N_{\phi}=-2 d \eta \otimes \xi
$$

where $N_{\phi}$ is the Nijenhuis tensor field of $\phi$ [4]. An almost contact metric manifold $N^{2 n+1}$ is called a Kenmotsu manifold [9] if

$$
\left(\nabla_{X} \phi\right) Y=g(\phi X, Y) \xi-\eta(Y) X
$$

where $\nabla$ is the Levi-Civita connection. An almost contact metric manifold $N^{2 n+1}$ is called a cosymplectic manifold if $\nabla \phi=0$, which implies that $\nabla \xi=0$ [13].

The sectional curvature of a $\phi$-section is called a $\phi$-sectional curvature. When the $\phi$-sectional curvature is constant, the manifold is called a space form (Sasakian,Kenmotsu, cosymplectic) (see [4], [9], [13]). The manifold $N^{2 n+1}$ $=\left(N^{2 n+1}, \varphi, \xi, \eta, g\right)$ is called a generalized Sasakian space form if its curvature tensor $R$ is given by

$$
\begin{gathered}
R(X, Y) Z=f_{1}\{g(Y, Z) X-g(X, Z) Y\} \\
+f_{2}\{g(X, \phi Z) \phi Y-g(Y, \phi Z) \phi X+2 g(X, \phi Y) \phi Z\} \\
+f_{3}\{\eta(X) \eta(Z) Y-\eta(X) \eta(Z) X+g(X, Z) \eta(Y) \xi-g(Y, Z) \eta(X) \xi\}
\end{gathered}
$$

for certain differentiable functions $f_{1}, f_{2}$ and $f_{3}$ on $N^{2 n+1}$ [1]. If $N^{2 n+1}$ is a Sasakian space form then $f_{1}=\frac{c+3}{4}$, $f_{2}=f_{3}=\frac{c-1}{4}$ [4], if $N^{2 n+1}$ is a Kenmotsu space form then $f_{1}=\frac{c-3}{4}, f_{2}=f_{3}=\frac{c+1}{4}$ [9], if $N^{2 n+1}$ is a cosymplectic space form then $f_{1}=f_{2}=f_{3}=\frac{c}{4}$ [13].

A submanifold of a Sasakian manifold is called an integral submanifold if $\eta(X)=0$, for every tangent vector $X$. An integral curve of a Sasakian manifold $\left(N^{2 n+1}, \phi, \xi, \eta, g\right)$ is called a Legendre curve [4]. Thus, a curve $\gamma: I \longrightarrow\left(N^{2 n+1}, \phi, \xi, \eta, g\right)$ is called a Legendre curve if $\eta(T)=0$, where $T$ is the tangent vector field of $\gamma$.

In [15], the notion of trans-Sasakian manifolds is introduced by Oubiña. An almost contact metric manifold $N$ is said to be a trans-Sasakian manifold if there exist two functions $\alpha$ and $\beta$ on $N$ such that

$$
\left(\nabla_{X} \phi\right) Y=\alpha[g(X, Y) \xi-\eta(Y) X]+\beta[g(\phi X, Y) \xi-\eta(Y) \phi X],
$$

for any vector fields $X, Y$ on $N$. From (2.2), it is easy to see that

$$
\nabla_{X} \xi=-\alpha \phi X+\beta[X-\eta(X) \xi] .
$$

If we have $\beta=0$ (resp. $\alpha=0$ ), then $N$ is called an $\alpha$-Sasakian manifold (resp. $\beta$-Kenmotsu manifold). Another kind of trans-Sasakian manifolds is that of cosymplectic manifolds, obtained for $\alpha=\beta=0$. By the use of the equation (2.3), we have

$$
\nabla_{X} \xi=0
$$

which means that $\xi$ is a Killing vector field for a cosymplectic manifold [3]. 


\section{Interpolating sesqui-harmonic Legendre curves in generalized Sasakian space forms}

Let $(N, g)$ be an $n$-dimensional Riemannian manifold and $\gamma: I \longrightarrow(N, g)$ a unit-speed curve in $(N, g)$. $\gamma$ is called a Frenet curve of osculating order $r, 1 \leq r \leq n$, if there exists orthonormal vector fields $\left\{E_{i}\right\}_{i=1,2, \ldots n}$ along $\gamma$ satisfying Frenet equations given by

$$
\begin{gathered}
E_{1}=T=\gamma^{\prime}, \\
\nabla_{T} E_{1}=k_{1} E_{2}, \\
\nabla_{T} E_{i}=-k_{i-1} E_{i-1}+k_{i} E_{i+1}, 2 \leq i \leq n-1, \\
\nabla_{T} E_{n}=-k_{n-1} E_{n-1},
\end{gathered}
$$

where the function $\left\{k_{1}=k, k_{2}=\tau, k_{3}, \ldots, k_{n-1}\right\}$ are called the curvatures of $\gamma$ [11].

Now, we can state the following theorem:

Theorem 3.1. Let $\left(N^{2 n+1}, \phi, \xi, \eta, g\right)$ be a generalized Sasakian space form with constant $\phi$-sectional curvature $c$ and $\gamma: I \subset \mathbb{R} \longrightarrow\left(N^{2 n+1}, \phi, \xi, \eta, g\right)$ be a Legendre curve of osculating order $r$ and $m=\min \{r, 4\}$. Then $\gamma$ is interpolating sesqui-harmonic if and only if there exists real numbers $\delta_{1}, \delta_{2}$ such that

(1) $f_{2}=0$ or $\phi T \perp E_{2}$ or $\phi T \in\left\{E_{2}, \ldots, E_{m}\right\}$; and

(2) $f_{3}=0$ or $\xi \perp E_{2}$ or $\xi \in\left\{E_{2}, \ldots, E_{m}\right\}$; and

(3) the first $m$ of the following equations are satisfied:

$$
\begin{gathered}
-3 \delta_{2} k_{1} k_{1}^{\prime}=0, \\
\delta_{2}\left(k_{1}^{\prime \prime}-k_{1}^{3}-k_{1} k_{2}^{2}-k_{1} f_{1}\right)-\delta_{1} k_{1}+3 \delta_{2} f_{2} k_{1}\left[g\left(\phi T, E_{2}\right)\right]^{2}-\delta_{2} f_{3} k_{1}\left[\eta\left(E_{2}\right)\right]^{2}=0, \\
\delta_{2}\left(2 k_{1}^{\prime} k_{2}+k_{1} k_{2}^{\prime}\right)+3 \delta_{2} f_{2} k_{1} g\left(\phi T, E_{2}\right) g\left(\phi T, E_{3}\right)-\delta_{2} f_{3} k_{1} \eta\left(E_{2}\right) \eta\left(E_{3}\right)=0, \\
\delta_{2}\left(k_{1} k_{2} k_{3}\right)+3 \delta_{2} f_{2} k_{1} g\left(\phi T, E_{2}\right) g\left(\phi T, E_{4}\right)-\delta_{2} f_{3} k_{1} \eta\left(E_{2}\right) \eta\left(E_{4}\right)=0 .
\end{gathered}
$$

Proof. Let $\left(N^{2 n+1}, \phi, \xi, \eta, g\right)$ be a generalized Sasakian space form and $\gamma: I \longrightarrow N^{2 n+1}$ be a Legendre curve of osculating order $r$. By the use of (1.1) and (3.1), we can write

$$
\tau(\gamma)=k_{1} E_{2}
$$

Using the equations (3.1), we find

$$
\begin{gathered}
\nabla_{T} \nabla_{T} T=-k_{1}^{2} E_{1}+k_{1}^{\prime} E_{2}+k_{1} k_{2} E_{3}, \\
\nabla_{T} \nabla_{T} \nabla_{T} T=-3 k_{1} k_{1}^{\prime} E_{1}+\left(k_{1}^{\prime \prime}-k_{1}^{3}-k_{1} k_{2}^{2}\right) E_{2} \\
+\left(2 k_{1}^{\prime} k_{2}+k_{1} k_{2}^{\prime}\right) E_{3}+\left(k_{1} k_{2} k_{3}\right) E_{4}, \\
R\left(T, \nabla_{T} T\right) T=-k_{1} f_{1} E_{2}-3 f_{2} k_{1} g\left(\phi T, E_{2}\right) \phi T+f_{3} k_{1} \eta\left(E_{2}\right) \xi .
\end{gathered}
$$

By the use of the equations (3.6), (3.7) and (3.8) into (4.1) in [6], we obtain

$$
\begin{gathered}
\tau_{\delta_{1}, \delta_{2}}(\gamma)=\left(-3 \delta_{2} k_{1} k_{1}^{\prime}\right) E_{1}+\left[\delta_{2}\left(k_{1}^{\prime \prime}-k_{1}^{3}-k_{1} k_{2}^{2}+f_{1} k_{1}\right)-\delta_{1} k_{1}\right] E_{2} \\
+\delta_{2}\left(2 k_{1}^{\prime} k_{2}+k_{1} k_{2}^{\prime}\right) E_{3}+\delta_{2}\left(k_{1} k_{2} k_{3}\right) E_{4}+3 \delta_{2} f_{2} k_{1} g\left(\phi T, E_{2}\right) \phi T-\delta_{2} f_{3} k_{1} \eta\left(E_{2}\right) \xi .
\end{gathered}
$$

Then taking the scalar product of (3.9) with $E_{2}, E_{3}$ and $E_{4}$ respectively, we obtain the desired results.

Now, we give the interpretions of Theorem 3.1:

Case I. $f_{2}=f_{3}=0$.

From Theorem 3.1, we obtain following theorem: 
Theorem 3.2. Let $\left(N^{2 n+1}, \phi, \xi, \eta, g\right)$ be a generalized Sasakian space form with $f_{2}=f_{3}=0$ and $\gamma: I \subset \mathbb{R} \longrightarrow N^{2 n+1}(1)$ be a Legendre curve of osculating order $r$.

(1) $\gamma$ is interpolating sesqui-harmonic with $\frac{\delta_{1}}{\delta_{2}} \neq 0$ if and only if it is a Legendre circle with $k_{1}=\sqrt{f_{1}-\frac{\delta_{1}}{\delta_{2}}}$ where $f_{1}>\frac{\delta_{1}}{\delta_{2}}$ is a constant;

(2) $\gamma$ is interpolating sesqui-harmonic with $\frac{\delta_{1}}{\delta_{2}} \neq 0$ if and only if it is a Legendre helix with $k_{1}^{2}+k_{2}^{2}=f_{1}-\frac{\delta_{1}}{\delta_{2}}$ where $f_{1}>\frac{\delta_{1}}{\delta_{2}}, \delta_{1}, \delta_{2}$ is a constant.

In both cases, if $f_{1} \leq \frac{\delta_{1}}{\delta_{2}}$, then such an interpolating sesqui-harmonic Legendre curve does not exist.

Proof. Let $\gamma: I \longrightarrow N^{2 n+1}$ be an interpolating sesqui-harmonic curve. From Theorem 3.1, if we take $r=2$, then $\gamma$ is a circle with $k_{1}=\sqrt{f_{1}-\frac{\delta_{1}}{\delta_{2}}}$ where $f_{1}>\frac{\delta_{1}}{\delta_{2}}$ is a constant. Similarly, if we take $r=3$, then we obtain that $k_{2}$ is a non-zero constant. Thus, $\gamma$ is a helix with $k_{1}^{2}+k_{2}^{2}=f_{1}-\frac{\delta_{1}}{\delta_{2}}$ where $f_{1}>\frac{\delta_{1}}{\delta_{2}}$ is a constant. On the contrary, let $\gamma$ be a Legendre circle with $k_{1}=\sqrt{f_{1}-\frac{\delta_{1}}{\delta_{2}}}$ or a Legendre helix with $k_{1}^{2}+k_{2}^{2}=f_{1}-\frac{\delta_{1}}{\delta_{2}}$ where $f_{1}>\frac{\delta_{1}}{\delta_{2}}$ is a constant. It is clear that $\gamma$ satisfies Theorem 3.1, respectively. Thus, we obtain the desired result.

Case II. $f_{2}=0, f_{3} \neq 0$ and $\xi \perp E_{2}$.

We can state:

Theorem 3.3. Let $\left(N^{2 n+1}, \phi, \xi, \eta, g\right)$ be a generalized Sasakian space form with $f_{2}=0, f_{3} \neq 0, \xi \perp E_{2}$ and $\gamma: I \subset \mathbb{R} \longrightarrow$ $N^{2 n+1}(c)$ a Legendre curve of osculating order $r$.

(1) $\gamma$ is interpolating sesqui-harmonic with $\frac{\delta_{1}}{\delta_{2}} \neq 0$ if and only if it is a Legendre circle with $k_{1}=\sqrt{f_{1}-\frac{\delta_{1}}{\delta_{2}}}$ where $f_{1}>\frac{\delta_{1}}{\delta_{2}}$ is a constant; or

(2) $\gamma$ is interpolating sesqui-harmonic with $\frac{\delta_{1}}{\delta_{2}} \neq 0$ if and only if it is a Legendre helix with $k_{1}^{2}+k_{2}^{2}=f_{1}-\frac{\delta_{1}}{\delta_{2}}$ where $f_{1}>\frac{\delta_{1}}{\delta_{2}}, \delta_{1}, \delta_{2}$ is a constant.

If $r>3$ or $f_{1} \leq \frac{\delta_{1}}{\delta_{2}}$, then an interpolating sesqui-harmonic Legendre curve does not exist.

Proof. Assume that $\gamma: I \longrightarrow N^{2 n+1}$ be an interpolating sesqui-harmonic curve. From Theorem 3.1 and $\eta\left(E_{2}\right)=0$, we have

$$
\begin{gathered}
k_{1}=\text { constant }>0, \\
k_{1}^{2}+k_{2}^{2}=f_{1}-\frac{\delta_{1}}{\delta_{2}}, \\
k_{2}^{\prime}=0 \\
k_{2} k_{3}=0 .
\end{gathered}
$$

Using the above equations, we obtain the desired results.

Case III. $f_{2}=0, f_{3} \neq 0, \xi \in \operatorname{span}\left\{E_{2}, \ldots, E_{m}\right\}$ and $\eta\left(E_{2}\right) \neq 0$.

Theorem 3.4. Let $\left(N^{2 n+1}, \phi, \xi, \eta, g\right)$ be a generalized Sasakian space form with $f_{2}=0, f_{3} \neq 0, \xi \in \operatorname{span}\left\{E_{2}, \ldots, E_{m}\right\}$, $\eta\left(E_{2}\right) \neq 0$ and $\gamma: I \subset \mathbb{R} \longrightarrow N^{2 n+1}(c)$ a Legendre curve of osculating order $r$.

(1) If $r \geq 4$, then $\gamma$ is interpolating sesqui-harmonic with $\frac{\delta_{1}}{\delta_{2}} \neq 0$ if and only if

$$
\begin{gathered}
k_{1}=\text { constant }>0, \\
k_{1}^{2}+k_{2}^{2}=f_{1}-\frac{\delta_{1}}{\delta_{2}}-f_{3} \cos ^{2} u_{1}, \\
k_{2}^{\prime}-f_{3} \cos u_{1} \sin u_{1} \cos u_{2}=0, \\
k_{2} k_{3}-f_{3} \cos u_{1} \sin u_{1} \sin u_{2}=0 .
\end{gathered}
$$

(2) If we take $r=3$, the equations (3.10), (3.11) and (3.12) are satisfied, taking $u_{2}=0$. If we take $r=2$, then the equations (3.10) and (3.11) are satisfied, taking $u_{1}=0, \pi$. 
Proof. Assume that $r \geq 4$. Thus, we can write

$$
\xi=\cos u_{1} E_{2}+\sin u_{1} \cos u_{2} E_{3}+\sin u_{1} \sin u_{2} E_{4}
$$

where $u_{1}, u_{2}: I \rightarrow \mathbb{R}$ are the angle functions between $\xi$ and $E_{2} ; E_{3}$ and the orthogonal projection of $\xi$ onto $\operatorname{span}\left\{E_{3}, E_{4}\right\}$, respectively. From the equation (3.13), we have

$$
\begin{gathered}
\eta\left(E_{2}\right)=\cos u_{1}, \\
\eta\left(E_{3}\right)=\sin u_{1} \cos u_{2}, \\
\eta\left(E_{4}\right)=\sin u_{1} \sin u_{2} .
\end{gathered}
$$

Assume that $r=3$. We can write

$$
\xi=\cos u_{1} E_{2}+\sin u_{1} E_{3}
$$

where $u_{1}: I \rightarrow \mathbb{R}$ is the angle function between $\xi$ and $E_{2}$. The equation (3.15) can be found taking $u_{2}=0$ in (3.13). Finally, let $r=2$. We can write

$$
\xi=\mp E_{2} .
$$

We obtain (3.16) from (3.13), taking $u_{1}=0, \pi$ and $u_{2}=0$. Using Theorem 3.1 and the equations (3.13), (3.15) and (3.16), we obtain the desired results.

Now, let $\gamma: I \subset \mathbb{R} \longrightarrow N^{2 n+1}$ be a Legendre curve of osculating order $r$ in trans-Sasakian generalized Sasakian space form $\left(N^{2 n+1}, \phi, \xi, \eta, g\right)$. Since $\gamma$ is a Legendre curve, $\eta(T)=0$. Then, we have

$$
\nabla_{T} \xi=-\alpha \phi T+\beta T
$$

which gives us

$$
g\left(\nabla_{T} \xi, T\right)=\beta .
$$

Differentiating $\eta(T)=0$ along $\gamma$, if we use (3.1) and (3.18), we get

$$
k_{1} \eta\left(E_{2}\right)=-\beta .
$$

Corollary 3.1. Let $\left(N^{2 n+1}, \phi, \xi, \eta, g\right)$ be a trans-Sasakian generalized Sasakian space form with $f_{1}=$ constant, $f_{2}=0, f_{3}$ and $\beta$ are non-zero constants, $\xi \in$ span $\left\{E_{2}, \ldots, E_{m}\right\}, \eta\left(E_{2}\right) \neq 0$ and $\gamma: I \subset \mathbb{R} \longrightarrow N^{2 n+1}$ a Legendre curve of osculating order $r$.

(1) $\gamma$ is interpolating sesqui-harmonic with $\frac{\delta_{1}}{\delta_{2}}<0$ if and only if $\gamma$ is a circle with $k_{1}=\sqrt{f_{1}-\frac{\delta_{1}}{\delta_{2}}-f_{3}}$ where $f_{1}-\frac{\delta_{1}}{\delta_{2}}-f_{3}>$ 0 is a constant, $0<\beta^{2}<-\frac{\delta_{1}}{\delta_{2}}, \xi \| E_{2}$ and $\alpha=0$, or

(2) $\gamma$ is interpolating sesqui-harmonic with $\frac{\delta_{1}}{\delta_{2}} \neq 0$ if and only if $\gamma$ is a helix with $k_{1}=\mp \beta>0, k_{2}=\sqrt{f_{1}-\frac{\delta_{1}}{\delta_{2}}-f_{3}-\beta^{2}}$, where $f_{1}-\frac{\delta_{1}}{\delta_{2}}-f_{3}-\beta^{2}>0$ is a constant, $\xi \| E_{2}, \alpha \neq 0$ is a constant, $\phi T \| E_{3}$ and $\operatorname{dim} N=3$.

Proof. Let $N^{2 n+1}$ be a trans-Sasakian generalized Sasakian space form, then we have

$$
\begin{gathered}
\nabla_{T} \eta\left(E_{1}\right)=k_{1} \eta\left(E_{2}\right)+\beta=0, \\
\nabla_{T} \eta\left(E_{2}\right)=k_{2} \eta\left(E_{3}\right)-\alpha g\left(\phi T, E_{2}\right), \\
\nabla_{T} \eta\left(E_{3}\right)=-k_{2} \eta\left(E_{2}\right)+k_{3} \eta\left(E_{4}\right)-\alpha g\left(\phi T, E_{3}\right), \\
\nabla_{T} \eta(E 4)=-k_{3} \eta\left(E_{3}\right)+k_{4} \eta\left(E_{5}\right)-\alpha g\left(\phi T, E_{4}\right) .
\end{gathered}
$$

Let $\gamma$ be interpolating sesqui-harmonic.

1. If $r=2$, from Theorem 3.1, we have

$$
\begin{gathered}
k_{1}=\text { constant }>0, \\
k_{1}^{2}=f_{1}-\frac{\delta_{1}}{\delta_{2}}-f_{3}\left[\eta\left(E_{2}\right)\right]^{2}
\end{gathered}
$$


and $\xi \in \operatorname{span}\left\{E_{2}\right\}$. Hence we obtain $\eta\left(E_{2}\right)= \pm 1$. So $\gamma$ is a circle with $k_{1}=\sqrt{f_{1}-\frac{\delta_{1}}{\delta_{2}}-f_{3}}$, where $f_{1}-\frac{\delta_{1}}{\delta_{2}}-f_{3}>0$ is a constant and $\xi \| E_{2}$. Differentiating $\xi= \pm E_{2}$ along $\gamma$, we get $\alpha=0$ and $k_{1}= \pm \beta$. Since $\alpha=0, N$ is a $\beta$-Kenmotsu generalized Sasakian space form. Then $\beta$-Kenmotsu generalized Sasakian space forms satisfy

$$
f_{1}-f_{3}+\beta^{2}=0 .
$$

Then, we have $0<\beta^{2}<-\frac{\delta_{1}}{\delta_{2}}$ with $\frac{\delta_{1}}{\delta_{2}}<0$.

2. If we take $r=3$, using Theorem 3.1, we have

$$
\begin{gathered}
k_{1}=\text { constant }>0, \\
k_{1}^{2}+k_{2}^{2}=f_{1}-\frac{\delta_{1}}{\delta_{2}}-f_{3}\left[\eta\left(E_{2}\right)\right]^{2}, \\
k_{2}^{\prime}-f_{3} \eta\left(E_{2}\right) \eta\left(E_{4}\right)=0
\end{gathered}
$$

and $\xi \in \operatorname{span}\left\{E_{2}, E_{4}\right\}$. Differentiating the equation (3.23) and using (3.21), (3.24), we get

$$
2 k_{2} \eta\left(E_{3}\right)=\alpha g\left(\phi T, E_{2}\right) .
$$

From the equation (3.20), we obtain that $\eta\left(E_{2}\right)$ is a constant, since $\beta \neq 0$ is a constant. Using (3.21), we find

$$
k_{2} \eta\left(E_{3}\right)=\alpha g\left(\phi T, E_{2}\right) .
$$

By the use of (3.25) and (3.26), we obtain $\eta\left(E_{3}\right)=0$. Since $\xi \in \operatorname{span}\left\{E_{2}, E_{3}\right\}$ and $\eta\left(E_{3}\right)=0$, we find $\xi \| E_{2}$. From the equations (3.20), (3.22), (3.23) and (3.24), we obtain that $\gamma$ is a helix with $k_{1}= \pm \beta>0, k_{2}=\sqrt{f_{1}-\frac{\delta_{1}}{\delta_{2}}-f_{3}-\beta^{2}}$, where $f_{1}-\frac{\delta_{1}}{\delta_{2}}-f_{3}-\beta^{2}>0$ is a constant and $\xi \| E_{2}$. Differentiating $\xi= \pm E_{2}$ along $\gamma$, we get $\alpha \neq 0$. From [14], we obtain $\operatorname{dim} \stackrel{\delta_{2}}{N}=3$.

3. If we take $r \geq 4$, then $\operatorname{dim} N \geq 5$. Since $\beta \neq 0$ is a constant, from [14], we have $\alpha=0$. Thus we obtain that $N$ is a $\beta$-Kenmotsu generalized Sasakian space form and $\operatorname{dim} N \geq 5$. Using [1], we find $f_{3}=0$, which is a contradiction.

On the contrary, let $\gamma$ be the given curve. It is easily seen that the first three of the equations in Theorem 3.1 are satisfied (replacing $k_{m}=0$ ). So $\gamma$ is interpolating sesqui-harmonic.

Case IV. $f_{2} \neq 0, f_{3}=0$ and $\phi T \perp E_{2}$.

In this case, we have $g\left(\phi T, E_{2}\right)=0$. From Theorem 3.1, we have

Theorem 3.5. Let $\left(N^{2 n+1}, \phi, \xi, \eta, g\right)$ be a generalized Sasakian space form with $f_{2} \neq 0, f_{3}=0, \phi T \perp E_{2}$ and $\gamma: I \subset$ $\mathbb{R} \longrightarrow N^{2 n+1}(c)$ a Legendre curve of osculating order $r$.

(1) $\gamma$ is interpolating sesqui-harmonic with $\frac{\delta_{1}}{\delta_{2}} \neq 0$ if and only if it is a Legendre circle with $k_{1}=\sqrt{f_{1}-\frac{\delta_{1}}{\delta_{2}}}$ where $f_{1}>\frac{\delta_{1}}{\delta_{2}}$ is a constant; or

(2) $\gamma$ is interpolating sesqui-harmonic with $\frac{\delta_{1}}{\delta_{2}} \neq 0$ if and only if it is a Legendre helix with $k_{1}^{2}+k_{2}^{2}=f_{1}-\frac{\delta_{1}}{\delta_{2}}$ where $f_{1}>\frac{\delta_{1}}{\delta_{2}}, \delta_{1}, \delta_{2}$ is a constant.

If $f_{1} \leq \frac{\delta_{1}}{\delta_{2}}$, then an interpolating sesqui-harmonic Legendre curve does not exist.

Case V. $f_{2} \neq 0, f_{3}=0, \phi T \in \operatorname{span}\left\{E_{2}, E_{3}, E_{4}\right\}$ and $g\left(\phi T, E_{2}\right) \neq 0$.

Theorem 3.6. Let $\left(N^{2 n+1}, \phi, \xi, \eta, g\right)$ be a generalized Sasakian space form with $f_{2} \neq 0, f_{3}=0, \phi T \in \operatorname{span}\left\{E_{2}, E_{3}, E_{4}\right\}$, $g\left(\phi T, E_{2}\right) \neq 0$ and $\gamma: I \subset \mathbb{R} \longrightarrow N^{2 n+1}(c)$ a Legendre curve of osculating order $r$.

(1) If $r \geq 4$, then $\gamma$ is interpolating sesqui-harmonic with $\frac{\delta_{1}}{\delta_{2}} \neq 0$ if and only if

$$
\begin{gathered}
k_{1}=\text { constant }>0, \\
k_{1}^{2}+k_{2}^{2}=f_{1}-\frac{\delta_{1}}{\delta_{2}}+3 f_{2} \cos ^{2} w_{1}, \\
k_{2}^{\prime}+3 f_{2} \cos w_{1} \sin w_{1} \cos w_{2}=0, \\
k_{2} k_{3}+3 f_{2} \cos w_{1} \sin w_{1} \sin w_{2}=0 .
\end{gathered}
$$

(2) If we take $r=3$, the equations (3.27), (3.28) and (3.29) are satisfied, taking $w_{2}=0$. If we take $r=2$, then the equations (3.27) and (3.28) are satisfied, taking $w_{1}=0, \pi$. 
Proof. Assume that $r \geq 4$. Thus, we can write

$$
\phi T=\cos w_{1} E_{2}+\sin w_{1} \cos w_{2} E_{3}+\sin w_{1} \sin w_{2} E_{4}
$$

where $w_{1}, w_{2}: I \rightarrow \mathbb{R}$ are the angle functions between $\phi T$ and $E_{2} ; E_{3}$ and the orthogonal projection of $\phi T$ onto $\operatorname{span}\left\{E_{3}, E_{4}\right\}$, respectively. From the equation (3.30), we can write

$$
\begin{gathered}
g\left(\phi T, E_{2}\right)=\cos w_{1}, \\
g\left(\phi T, E_{3}\right)=\sin w_{1} \cos w_{2}, \\
g\left(\phi T, E_{4}\right)=\sin w_{1} \sin w_{2} .
\end{gathered}
$$

Let $r=3$. We can write

$$
\phi T=\cos w_{1} E_{2}+\sin w_{1} E_{3}
$$

where $w_{1}: I \rightarrow \mathbb{R}$ is the angle function between $\phi T$ and $E_{2}$. The equation (3.32) can be found taking $w_{2}=0$ in (3.30). Finally, let $r=2$. We can write

$$
\phi T=\mp E_{2} .
$$

We obtain (3.33) from (3.30), taking $w_{1}=0, \pi$ and $w_{2}=0$. Using Theorem 3.1 and the equations (3.30), (3.32) and (3.33), we obtain the desired results.

Using the same method of Corollary 3.2 in [16], we have the following corollary:

Corollary 3.2. Let $\left(N^{2 n+1}, \phi, \xi, \eta, g\right)$ be a connected trans-Sasakian generalized Sasakian space form with $f_{1}=$ constant, $f_{2} \neq 0$ is a constant, $f_{3}=0, \phi T \in \operatorname{span}\left\{E_{2}, \ldots, E_{m}\right\}, g\left(\phi T, E_{2}\right) \neq 0$ and $\gamma: I \subset \mathbb{R} \longrightarrow N^{2 n+1}$ a Legendre curve of osculating order $r$. Then $\gamma$ is interpolating sesqui-harmonic with $\frac{\delta_{1}}{\delta_{2}} \neq 0$ if and only if $\gamma$ is a Frenet curve of order $r \geq 4$ with

$$
\begin{gathered}
k_{1}=\frac{-\beta}{\eta\left(E_{2}\right)}=\text { constant }>0, \\
k_{2}=\sqrt{f_{1}-\frac{\delta_{1}}{\delta_{2}}+3 f_{2}\left[g\left(\phi T, E_{2}\right)\right]^{2}-\frac{\beta^{2}}{\left[\eta\left(E_{2}\right)\right]^{2}}}>0, \\
k_{3}=\frac{-3 g\left(\phi T, E_{2}\right) g\left(\phi T, E_{4}\right)}{\sqrt{f_{1}-\frac{\delta_{1}}{\delta_{2}}+3 f_{2}\left[g\left(\phi T, E_{2}\right)\right]^{2}-\frac{\beta^{2}}{\left[\eta\left(E_{2}\right)\right]^{2}}}}>0, \\
k_{4}=\frac{-\beta g\left(\phi E_{2}, E_{5}\right)}{\eta\left(E_{2}\right) g\left(\phi T, E_{4}\right)}>0, i f r \geq 5
\end{gathered}
$$

where $f_{1}-\frac{\delta_{1}}{\delta_{2}}+3 f_{2}\left[g\left(\phi T, E_{2}\right)\right]^{2}-\frac{\beta^{2}}{\left[\eta\left(E_{2}\right)\right]^{2}}$ is a constant, $g\left(\phi T, E_{3}\right)=0, \alpha=0, g\left(\phi T, E_{2}\right) \neq 0$ and $g\left(\phi T, E_{4}\right) \neq 0$ are constants, $\beta \neq 0$ and $\eta\left(E_{2}\right) \neq 0$.

Proof. Assume that $N^{2 n+1}$ is a trans-Sasakian generalized Sasakian space form, then we have

$$
\begin{gathered}
\nabla_{T} \phi T=\alpha \xi+k_{1} \phi E_{2}, \\
\nabla_{T} g\left(\phi T, E_{2}\right)=\alpha \eta\left(E_{2}\right)+k_{2} g\left(\phi T, E_{3}\right), \\
\nabla_{T} g\left(\phi T, E_{3}\right)=\alpha \eta\left(E_{3}\right)+k_{1} g\left(\phi E_{2}, E_{3}\right)-k_{2} g\left(\phi T, E_{2}\right)+k_{3} g\left(\phi T, E_{4}\right), \\
\nabla_{T} g\left(\phi T, E_{4}\right)=\alpha \eta\left(E_{4}\right)+k_{2} g\left(\phi E_{2}, E_{4}\right)-k_{3} g\left(\phi T, E_{3}\right) .
\end{gathered}
$$

Let $\gamma$ be interpolating sesqui-harmonic.

1. If we take $r=2$, using Theorem 3.1, we have

$$
\begin{gathered}
k_{1}=\text { constant }>0, \\
k_{1}^{2}=f_{1}-\frac{\delta_{1}}{\delta_{2}}+3 f_{2}\left[g\left(\phi T, E_{2}\right)\right]^{2}
\end{gathered}
$$


and $\phi T \in \operatorname{span}\left\{E_{2}\right\}$. Thus, we can write $\phi T= \pm E_{2}$. Differentiating $\phi T= \pm E_{2}$, using (3.1) and (3.34), we have

$$
\alpha \xi+k_{1} \phi E_{2}=\mp k_{1} T \text {. }
$$

Hence we obtain $\alpha=0$. From the equation (3.20), we get $\beta=0$. So $N$ is cosymplectic which requires $f_{2}=f_{3}$. This is a contradiction.

2. If $r=3$, from Theorem 3.1, we have

$$
\begin{gathered}
k_{1}=\text { constant }>0, \\
k_{1}^{2}+k_{2}^{2}=f_{1}-\frac{\delta_{1}}{\delta_{2}}+3 f_{2}\left[g\left(\phi T, E_{2}\right)\right]^{2}, \\
k_{2}^{\prime}+3 f_{2} g\left(\phi T, E_{2}\right) g\left(\phi T, E_{3}\right)=0
\end{gathered}
$$

and $\phi T \in \operatorname{span}\left\{E_{2}, E_{4}\right\}$. Differentiating the equation (3.38) and using (3.35), (3.39), we obtain

$$
-2 k_{2} g\left(\phi T, E_{3}\right)=\alpha \eta\left(E_{2}\right) \text {. }
$$

For $\phi T \in \operatorname{span}\left\{E_{2}, E_{4}\right\}$, we can write

$$
\phi T=g\left(\phi T, E_{2}\right) E_{2}+g\left(\phi T, E_{3}\right) E_{3} .
$$

It is clear that $g\left(\phi E_{2}, E_{3}\right)=0$. Differentiating the equation (3.41) and using (3.1), (3.34), (3.35) and (3.36), we find

$$
\alpha \xi+k_{1} \phi E_{2}=-k_{1} g\left(\phi T, E_{2}\right) T+\alpha \eta\left(E_{2}\right) E_{2}+\alpha \eta\left(E_{3}\right) E_{3} .
$$

Let $\alpha=0$. From the equation (3.42), we have $g\left(\phi T, E_{3}\right)=0$, that is, $\phi T= \pm E_{2}$. Using the equation (3.36), we find $k_{2}=0$. This is a contradiction. Thus $\alpha \neq 0$. From (3.42), we obtain

$$
\left[\eta\left(E_{2}\right)\right]^{2}+\left[\eta\left(E_{3}\right)\right]^{2}=1
$$

So $\xi \in \operatorname{span}\left\{E_{2}, E_{3}\right\}$ and $\phi T= \pm E_{2}$. Hence $\xi= \pm E_{3}$. Using the equation (3.20), we find $\beta=0$. Differentiating $\xi= \pm E_{3}$ and using the equations (3.1), (3.17), (3.37), (3.38) and (3.39), we obtain $k_{1}=\sqrt{f_{1}-\frac{\delta_{1}}{\delta_{2}}+3 f_{2}-\alpha^{2}}$, $k_{2}= \pm \alpha>0$, where $f_{1}-\frac{\delta_{1}}{\delta_{2}}+3 f_{2}-\alpha^{2}>0, \alpha \neq 0$ is a constant. Thus $N$ is a connected $\alpha$-Sasakian generalized Sasakian space form. If $\operatorname{dim} N \geq 5$, using [2], we find $f_{2}=f_{3}$, which is a contradiction. If $\operatorname{dim} N=3$, using [2], we have $f_{2}=0$, which is also a contradiction.

Assume that $r \geq 4$. From Theorem 3.1, we have

$$
\begin{gathered}
k_{1}=\text { constant }>0, \\
k_{1}^{2}+k_{2}^{2}=f_{1}-\frac{\delta_{1}}{\delta_{2}}+3 f_{2}\left[g\left(\phi T, E_{2}\right)\right]^{2}, \\
k_{2}^{\prime}+3 f_{2} g\left(\phi T, E_{2}\right) g\left(\phi T, E_{3}\right)=0, \\
k_{2} k_{3}+3 f_{2} g\left(\phi T, E_{2}\right) g\left(\phi T, E_{4}\right)=0
\end{gathered}
$$

and $\phi T \in \operatorname{span}\left\{E_{2}, E_{3}, E_{4}\right\}$. Differentiating (3.44) and using (3.35), (3.45), we get

$$
-2 k_{2} g\left(\phi T, E_{3}\right)=\alpha \eta\left(E_{2}\right)
$$

3. Assume that $r \geq 4$ and $g\left(\phi T, E_{3}\right)=0$. We obtain $\alpha=0$. Since $g\left(\phi T, E_{3}\right)=0$, we get $\phi T \in \operatorname{span}\left\{E_{2}, E_{4}\right\}$. By the use of equation (3.35), $g\left(\phi T, E_{2}\right) \neq 0$ is a constant. So using $\phi T \in \operatorname{span}\left\{E_{2}, E_{4}\right\}$ and (3.46), $g\left(\phi T, E_{4}\right) \neq 0$ is a constant. Using the equations (3.20), (3.43), (3.44), (3.45) and (3.46), we find $k_{1}=\frac{-\beta}{\eta\left(E_{2}\right)}=$ constant>

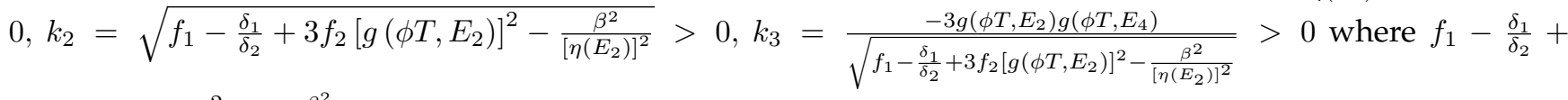
$3 f_{2}\left[g\left(\phi T, E_{2}\right)\right]^{2}-\frac{\beta^{2}}{\left[\eta\left(E_{2}\right)\right]^{2}}=$ constant $>0$. If $r \geq 5$, differentiating $g\left(\phi T, E_{5}\right)=0$ and using (3.34), we find $k_{4}=\frac{-\beta g\left(\phi E_{2}, E_{5}\right)}{\eta\left(E_{2}\right) g\left(\phi T, E_{4}\right)}$.

4. If $r \geq 4$ and $g\left(\phi T, E_{3}\right) \neq 0$, then $\alpha \neq 0$ and $\eta\left(E_{2}\right) \neq 0$. Since $\operatorname{dim} N \geq 5$ and $\alpha \neq 0$, we find $\beta=0$. This contradicts $\eta\left(E_{2}\right) \neq 0$.

On the contrary, let $\gamma$ be the given curve. Using Theorem $3.1, \gamma$ is interpolating sesqui-harmonic. 
Case VI. $f_{2} \neq 0, f_{3} \neq 0, \phi T \perp E_{2}$ and $\xi \perp E_{2}$.

In this case, we have $g\left(\phi T, E_{2}\right)=0$ and $\eta\left(E_{2}\right)=0$. Using Theorem 3.1, we have

Theorem 3.7. Let $\left(N^{2 n+1}, \phi, \xi, \eta, g\right)$ be a generalized Sasakian space form with $f_{2} \neq 0, f_{3} \neq 0, \phi T \perp E_{2}, \xi \perp E_{2}$ and $\gamma: I \subset \mathbb{R} \longrightarrow N^{2 n+1}(c)$ a Legendre curve of osculating order $r$.

(1) $\gamma$ is interpolating sesqui-harmonic with $\frac{\delta_{1}}{\delta_{2}} \neq 0$ if and only if it is a Legendre circle with $k_{1}=\sqrt{f_{1}-\frac{\delta_{1}}{\delta_{2}}}$ where $f_{1}>\frac{\delta_{1}}{\delta_{2}}$ is a constant; or

(2) $\gamma$ is interpolating sesqui-harmonic with $\frac{\delta_{1}}{\delta_{2}} \neq 0$ if and only if it is a Legendre helix with $k_{1}^{2}+k_{2}^{2}=f_{1}-\frac{\delta_{1}}{\delta_{2}}$ where $f_{1}>\frac{\delta_{1}}{\delta_{2}}, \delta_{1}, \delta_{2}$ is a constant.

If $f_{1} \leq \frac{\delta_{1}}{\delta_{2}}$, then an interpolating sesqui-harmonic Legendre curve does not exist.

Case VII. $f_{2} \neq 0, f_{3} \neq 0, \phi T \perp E_{2}, \xi \in \operatorname{span}\left\{E_{2}, \ldots, E_{m}\right\}$ and $\eta\left(E_{2}\right) \neq 0$.

Since $g\left(\phi T, E_{2}\right)=0$, using Theorem 3.1 and equations (3.13) and (3.14), we have

Theorem 3.8. Let $\left(N^{2 n+1}, \phi, \xi, \eta, g\right)$ be a generalized Sasakian space form with $f_{2} \neq 0, f_{3} \neq 0, \phi T \perp E_{2}, \xi \in$ $\operatorname{span}\left\{E_{2}, \ldots, E_{m}\right\}, \eta\left(E_{2}\right) \neq 0$. and $\gamma: I \subset \mathbb{R} \longrightarrow N^{2 n+1}(c)$ a Legendre curve of osculating order $r$.

(1) If $r \geq 4$, then $\gamma$ is interpolating sesqui-harmonic with $\frac{\delta_{1}}{\delta_{2}} \neq 0$ if and only if

$$
\begin{gathered}
k_{1}=\text { constant }>0, \\
k_{1}^{2}+k_{2}^{2}=f_{1}-\frac{\delta_{1}}{\delta_{2}}-f_{3} \cos ^{2} u_{1}, \\
k_{2}^{\prime}-f_{3} \cos u_{1} \sin u_{1} \cos u_{2}=0, \\
k_{2} k_{3}-f_{3} \cos u_{1} \sin u_{1} \sin u_{2}=0 .
\end{gathered}
$$

(2) If we take $r=3$, the equations (3.48), (3.49) and (3.50) are satisfied, taking $u_{2}=0$. If we take $r=2$, then the equations (3.48) and (3.49) are satisfied, taking $u_{1}=0, \pi$.

Corollary 3.3. Let $\left(N^{2 n+1}, \phi, \xi, \eta, g\right)$ be a trans-Sasakian generalized Sasakian space form with $f_{1}=$ constant, $f_{2}$ and $f_{3}$ are non-zero constants, $\phi T \perp E_{2}, \xi \in \operatorname{span}\left\{E_{2}, \ldots, E_{m}\right\}, \eta\left(E_{2}\right) \neq 0$ and $\gamma: I \subset \mathbb{R} \longrightarrow N^{2 n+1}$ a Legendre curve of osculating order $r$. Then $\gamma$ is interpolating sesqui-harmonic if and only if $\gamma$ is a helix of order $r \geq 4$ with

$$
\begin{gathered}
k_{1}=\frac{-\beta}{\eta\left(E_{2}\right)}=\text { constant }>0, \\
k_{2}=\sqrt{f_{1}-\frac{\delta_{1}}{\delta_{2}}-f_{3}\left[\eta\left(E_{2}\right)\right]^{2}-\frac{\beta^{2}}{\left[\eta\left(E_{2}\right)\right]^{2}}}>0, \\
k_{3}=\frac{f_{3} \eta\left(E_{2}\right) \eta\left(E_{4}\right)}{\sqrt{f_{1}-\frac{\delta_{1}}{\delta_{2}}-f_{3}\left[\eta\left(E_{2}\right)\right]^{2}-\frac{\beta^{2}}{\left[\eta\left(E_{2}\right)\right]^{2}}}}=\text { constant }>0,
\end{gathered}
$$

where $f_{1}-\frac{\delta_{1}}{\delta_{2}}-f_{3}\left[\eta\left(E_{2}\right)\right]^{2}-\frac{\beta^{2}}{\left[\eta\left(E_{2}\right)\right]^{2}}$ is a positive constant, $\eta\left(E_{3}\right)=0, \alpha=0$.

Proof. The proof is similar to the proof of Corollary 3.1.

Case VIII. $f_{2} \neq 0, f_{3} \neq 0, \phi T \perp E_{2}, \phi T \in \operatorname{span}\left\{E_{2}, \ldots, E_{m}\right\}, g\left(\phi T, E_{2}\right) \neq 0$ and $\xi \perp E_{2}$.

Since $\eta\left(E_{2}\right)=0$, using Theorem 3.1 and equations (3.30) and (3.31), we obtain the following theorem:

Theorem 3.9. Let $\left(N^{2 n+1}, \phi, \xi, \eta, g\right)$ be a generalized Sasakian space form with $f_{2} \neq 0, f_{3} \neq 0, \phi T \perp E_{2}, \phi T \in$ $\operatorname{span}\left\{E_{2}, \ldots, E_{m}\right\}, g\left(\phi T, E_{2}\right) \neq 0, \xi \perp E_{2}$ and $\gamma: I \subset \mathbb{R} \longrightarrow N^{2 n+1}(c)$ a Legendre curve of osculating order $r$.

(1) If $r \geq 4$, then $\gamma$ is interpolating sesqui-harmonic with $\frac{\delta_{1}}{\delta_{2}} \neq 0$ if and only if

$$
\begin{gathered}
k_{1}=\text { constant }>0, \\
k_{1}^{2}+k_{2}^{2}=f_{1}-\frac{\delta_{1}}{\delta_{2}}+3 f_{2} \cos ^{2} w_{1}, \\
k_{2}^{\prime}+3 f_{2} \cos w_{1} \sin w_{1} \cos w_{2}=0, \\
k_{2} k_{3}+3 f_{2} \cos w_{1} \sin w_{1} \sin w_{2}=0 .
\end{gathered}
$$

(2) If we take $r=3$, the equations (3.51), (3.52) and (3.53) are satisfied, taking $w_{2}=0$. If we take $r=2$, then the equations (3.51) and (3.52) are satisfied, taking $w_{1}=0, \pi$. 
Corollary 3.4. Let $\left(N^{2 n+1}, \phi, \xi, \eta, g\right)$ be a trans-Sasakian generalized Sasakian space form with $f_{1}=$ constant, $f_{2}$ and $f_{3}$ are non-zero constants, $\phi T \in \operatorname{span}\left\{E_{2}, \ldots, E_{m}\right\}, g\left(\phi T, E_{2}\right) \neq 0, \xi \perp E_{2}$ and $\gamma: I \subset \mathbb{R} \longrightarrow N^{2 n+1}$ a Legendre curve of osculating order $r$. Then $\gamma$ is interpolating sesqui-harmonic with $\frac{\delta_{1}}{\delta_{2}} \neq 0$ if and only if

(1) $\gamma$ is a circle with $k_{1}=\sqrt{f_{1}-\frac{\delta_{1}}{\delta_{2}}+3 f_{2}}$ where $\alpha=\beta=0, \phi T \| E_{2}$ and $f_{1}-\frac{\delta_{1}}{\delta_{2}}+3 f_{2}$ is a positive constant; or

(2) $\gamma$ is a helix with $k_{1}=\sqrt{f_{1}-\frac{\delta_{1}}{\delta_{2}}+3 f_{2}-\alpha^{2}}, k_{1}=\mp \alpha>0$ where $f_{1}-\frac{\delta_{1}}{\delta_{2}}+3 f_{2} \beta-\alpha^{2}>0, \alpha \neq 0$ is a constant, $\beta=0, \phi T \| E_{2}$ and $\xi \| E_{3}$; or

(3) $\gamma$ is a Frenet curve of order $r \geq 4$ with

$$
\begin{gathered}
k_{1}=\lambda>0, \\
k_{2}=\sqrt{f_{1}-\frac{\delta_{1}}{\delta_{2}}+3 f_{2}\left[g\left(\phi T, E_{2}\right)\right]^{2}-\lambda^{2}}>0, \\
k_{3}=\frac{-3 f_{2} g\left(\phi T, E_{2}\right) g\left(\phi T, E_{4}\right)}{\sqrt{f_{1}-\frac{\delta_{1}}{\delta_{2}}+3 f_{2}\left[g\left(\phi T, E_{2}\right)\right]^{2}-\lambda^{2}}}>0, \\
k_{4}=\frac{\lambda g\left(\phi E_{2}, E_{5}\right)}{g\left(\phi T, E_{4}\right)}>0, \text { if } r \geq 5
\end{gathered}
$$

where $g\left(\phi T, E_{3}\right)=0, g\left(\phi T, E_{2}\right) \neq 0$ and $g\left(\phi T, E_{4}\right) \neq 0$ are constants, $f_{1}-\frac{\delta_{1}}{\delta_{2}}+3 f_{2}\left[g\left(\phi T, E_{2}\right)\right]^{2}-\lambda^{2}>0$ and $\lambda>0$ are constants.

Proof. The proof is similar to the proof of Corollary 3.2.

Case IX. $f_{2} \neq 0, f_{3} \neq 0, \phi T \perp E_{2}, \phi T \in \operatorname{span}\left\{E_{2}, \ldots, E_{m}\right\}, g\left(\phi T, E_{2}\right) \neq 0$ and $\xi \in \operatorname{span}\left\{E_{2}, \ldots, E_{m}\right\}$ and $\eta\left(E_{2}\right) \neq 0$.

From Theorem 3.1 and equations (3.13), (3.14), (3.30) and (3.31), we have the following theorem:

Theorem 3.10. Let $\left(N^{2 n+1}, \phi, \xi, \eta, g\right)$ be a generalized Sasakian space form with $\phi T \in \operatorname{span}\left\{E_{2}, \ldots, E_{m}\right\}, \xi \in \operatorname{span}\left\{E_{2}, \ldots, E_{m}\right\}$ and $\gamma: I \subset \mathbb{R} \longrightarrow N^{2 n+1}(c)$ a Legendre curve of osculating order $r$.

(1) If $r \geq 4$, then $\gamma$ is interpolating sesqui-harmonic with $\frac{\delta_{1}}{\delta_{2}} \neq 0$ if and only if

$$
\begin{gathered}
k_{1}=\text { constant }>0, \\
k_{1}^{2}+k_{2}^{2}=f_{1}-\frac{\delta_{1}}{\delta_{2}}+3 f_{2} \cos ^{2} w_{1}-f_{3} \cos ^{2} u_{1}, \\
k_{2}^{\prime}+3 f_{2} \cos w_{1} \sin w_{1} \cos w_{2}-f_{3} \cos u_{1} \sin u_{1} \cos u_{2}=0, \\
k_{2} k_{3}+3 f_{2} \cos w_{1} \sin w_{1} \sin w_{2}-f_{3} \cos u_{1} \sin u_{1} \sin u_{2}=0 .
\end{gathered}
$$

(2) If we take $r=3$, the equations (3.54), (3.55) and (3.56) are satisfied, taking $w_{2}=0$ and $u_{2}=0$. If we take $r=2$, then the equations (3.54) and (3.55) are satisfied, taking $w_{1}=0, \pi$ and $u_{2}=0, \pi \cdot w_{1}=0, \pi$.

Corollary 3.5. Let $\left(N^{2 n+1}, \phi, \xi, \eta, g\right)$ be a trans-Sasakian generalized Sasakian space form with $f_{1}=$ constant, $f_{2}$ and $f_{3}$ are non-zero constants, $\phi T \in \operatorname{span}\left\{E_{2}, \ldots, E_{m}\right\}, g\left(\phi T, E_{2}\right) \neq 0, \xi \in \operatorname{span}\left\{E_{2}, \ldots, E_{m}\right\}, \eta\left(E_{2}\right) \neq 0$ and $\gamma: I \subset \mathbb{R} \longrightarrow$ $N^{2 n+1}$ a Legendre curve of osculating order $r \geq 4$. Then $\gamma$ is interpolating sesqui-harmonic with $\frac{\delta_{1}}{\delta_{2}} \neq 0$ if and only if

$$
\begin{gathered}
k_{1}=\frac{-\beta}{\eta\left(E_{2}\right)}=\text { constant }>0, \\
k_{2}=\frac{\lambda}{2 \mu}>0, \\
k_{3}=\frac{2 \mu\left\{f_{3} \eta\left(E_{2}\right) \eta\left(E_{4}\right)-3 f_{2} g\left(\phi T, E_{2}\right) g\left(\phi T, E_{4}\right)\right\}}{\lambda}>0,
\end{gathered}
$$

where $\lambda \neq 0$ and $\mu \neq 0$; or

(2)

$$
k_{1}=\frac{-\beta}{\eta\left(E_{2}\right)}=\text { constant }>0
$$




$$
\begin{gathered}
k_{2}=\sqrt{f_{1}-\frac{\delta_{1}}{\delta_{2}}+3 f_{2}\left[g\left(\phi T, E_{2}\right)\right]^{2}-f_{3}\left[\eta\left(E_{2}\right)\right]^{2}-\frac{\beta^{2}}{\left[\eta\left(E_{2}\right)\right]^{2}}}>0, \\
k_{3}=\frac{\left\{f_{3} \eta\left(E_{2}\right) \eta\left(E_{4}\right)-3 f_{2} g\left(\phi T, E_{2}\right) g\left(\phi T, E_{4}\right)\right\}}{\sqrt{f_{1}-\frac{\delta_{1}}{\delta_{2}}+3 f_{2}\left[g\left(\phi T, E_{2}\right)\right]^{2}-f_{3}\left[\eta\left(E_{2}\right)\right]^{2}-\frac{\beta^{2}}{\left[\eta\left(E_{2}\right)\right]^{2}}}}>0,
\end{gathered}
$$

where $f_{1}-\frac{\delta_{1}}{\delta_{2}}+3 f_{2}\left[g\left(\phi T, E_{2}\right)\right]^{2}-f_{3}\left[\eta\left(E_{2}\right)\right]^{2}-\frac{\beta^{2}}{\left[\eta\left(E_{2}\right)\right]^{2}}$ is a positive constant, $\lambda=\mu=0, \lambda=\left(3 f_{2}-f_{3}\right) \alpha g\left(\phi T, E_{2}\right) \eta\left(E_{2}\right)$ and $\mu=f_{3} \eta\left(E_{2}\right) \eta\left(E_{3}\right)-3 f_{2} g\left(\phi T, E_{2}\right) g\left(\phi T, E_{3}\right)$.

Proof. By the use of Theorem 3.1, we have

$$
\begin{gathered}
k_{1}=\text { constant }>0, \\
k_{1}^{2}+k_{2}^{2}=f_{1}-\frac{\delta_{1}}{\delta_{2}}+3 f_{2}\left[g\left(\phi T, E_{2}\right)\right]^{2}-f_{3}\left[\eta\left(E_{2}\right)\right]^{2}, \\
k_{2}^{\prime}-f_{3} \eta\left(E_{2}\right) \eta\left(E_{3}\right)+3 f_{2} g\left(\phi T, E_{2}\right) g\left(\phi T, E_{3}\right)=0, \\
k_{2} k_{3}-f_{3} \eta\left(E_{2}\right) \eta\left(E_{4}\right)+3 f_{2} g\left(\phi T, E_{2}\right) g\left(\phi T, E_{4}\right)=0 .
\end{gathered}
$$

Differentiating $\eta(T)=0$ along $\gamma$ and using (3.1), we obtain $k_{1} \eta\left(E_{2}\right)=-\beta$, that is,

$$
k_{1}=\frac{-\beta}{\eta\left(E_{2}\right)} .
$$

Differentiating the equation (3.57) along $\gamma$, we find

$$
k_{2} k_{2}^{\prime}=3 f_{2} g\left(\phi T, E_{2}\right) \nabla_{T} g\left(\phi T, E_{2}\right)-f_{3} \eta\left(E_{2}\right) \nabla_{T} \eta\left(E_{2}\right) .
$$

Since $N$ is a trans- Sasakian manifold, if we replace (3.21), (3.35), (3.58) in (3.60), we have

$$
2 k_{2} \mu=\lambda
$$

If $\lambda \neq 0$ and $\mu \neq 0$, then (3.61) gives us $k_{2}=\frac{\lambda}{2 \mu} \neq 0$. Thus, the equation (3.59) gives us $k_{3}$. If $\mu=0$, from the equation (3.58) that $k_{2}$ is a constant. Using the equation (3.57), we obtain $k_{2}=\sqrt{f_{1}-\frac{\delta_{1}}{\delta_{2}}+3 f_{2}\left[g\left(\phi T, E_{2}\right)\right]^{2}-f_{3}\left[\eta\left(E_{2}\right)\right]^{2}-\frac{\beta^{2}}{\left[\eta\left(E_{2}\right)\right]^{2}}}$, where $f_{1}-\frac{\delta_{1}}{\delta_{2}}+3 f_{2}\left[g\left(\phi T, E_{2}\right)\right]^{2}-f_{3}\left[\eta\left(E_{2}\right)\right]^{2}-\frac{\beta^{2}}{\left[\eta\left(E_{2}\right)\right]^{2}}>0$ is a constant. So the equation (3.59) gives us $k_{3}=\frac{\left\{f_{3} \eta\left(E_{2}\right) \eta\left(E_{4}\right)-3 f_{2} g\left(\phi T, E_{2}\right) g\left(\phi T, E_{4}\right)\right\}}{\sqrt{f_{1}-\frac{\delta_{1}}{\delta_{2}}+3 f_{2}\left[g\left(\phi T, E_{2}\right)\right]^{2}-f_{3}\left[\eta\left(E_{2}\right)\right]^{2}-\frac{\beta^{2}}{\left[\eta\left(E_{2}\right)\right]^{2}}}}>0$.

\section{Applications}

Let $\left(N^{2 n+1}, \phi, \xi, \eta, g\right)$ be a Sasakian space form. Thus we have $\alpha=1, \beta=0, f_{1}=\frac{c+3}{4}, f_{2}=f_{3}=\frac{c-1}{4}$. In this case, equation (3.19) gives us $\eta\left(E_{2}\right)=0$, since $k_{1}>0$.

For Case I and Case VI, using Theorem 3.2 and Theorem 3.7, then we obtain the following result in [10]:

Theorem 4.1. [10]Let $\left(N^{2 n+1}, \phi, \xi, \eta, g\right)$ be a Sasakian space form with $c=1$ or $\phi T \perp E_{2}$ and $\gamma: I \subset \mathbb{R} \longrightarrow N^{2 n+1} a$ Legendre curve of osculating order $r$. or

(1) $\gamma$ is interpolating sesqui-harmonic with $\frac{\delta_{1}}{\delta_{2}} \neq 0$ if and only if it is a Legendre circle with $k_{1}=\sqrt{1-\frac{\delta_{1}}{\delta_{2}}}$ where $1>\frac{\delta_{1}}{\delta_{2}}$;

(2) $\gamma$ is interpolating sesqui-harmonic with $\frac{\delta_{1}}{\delta_{2}} \neq 0$ if and only if it is a Legendre helix with $k_{1}^{2}+k_{2}^{2}=1-\frac{\delta_{1}}{\delta_{2}}$ where $1>\frac{\delta_{1}}{\delta_{2}}$.

If $1 \leq \frac{\delta_{1}}{\delta_{2}}$, then an interpolating sesqui-harmonic Legendre curve does not exist.

For Case VIII, if we use Corollary 3.4, we obtain the following theorem: 
Theorem 4.2. Let $\left(N^{2 n+1}, \phi, \xi, \eta, g\right)$ be a Sasakian space form with $c \neq 1, \phi T \in\left\{E_{2}, \ldots, E_{m}\right\}, g\left(\phi T, E_{2}\right) \neq 0$ and $\gamma: I \subset \mathbb{R} \longrightarrow N^{2 n+1}$ a Legendre curve of osculating order $r$.

(1) $\gamma$ is interpolating sesqui-harmonic with $\frac{\delta_{1}}{\delta_{2}} \neq 0$ if and only if it is a helix with $k_{1}=\sqrt{c-1-\frac{\delta_{1}}{\delta_{2}}}$ and $k_{2}=1$ where $c>\frac{\delta_{1}}{\delta_{2}}+1, \phi T \| E_{2}$ and $\xi \| E_{3}$; or

(2) $\gamma$ is interpolating sesqui-harmonic with $\frac{\delta_{1}}{\delta_{2}} \neq 0$ if and only if it is a Frenet curve of order $r \geq 4$ with

$$
\begin{gathered}
k_{1}=\lambda>0, \\
k_{2}=\sqrt{\frac{c+3}{4}-\frac{\delta_{1}}{\delta_{2}}+\frac{3(c-1)}{4}\left[g\left(\phi T, E_{2}\right)\right]^{2}-\lambda^{2}}>0, \\
k_{3}=\frac{-\frac{3(c-1)}{4} g\left(\phi T, E_{2}\right) g\left(\phi T, E_{4}\right)}{\sqrt{\frac{c+3}{4}-\frac{\delta_{1}}{\delta_{2}}+\frac{3(c-1)}{4}\left[g\left(\phi T, E_{2}\right)\right]^{2}-\lambda^{2}}}>0, \\
k_{4}=\frac{\lambda g\left(\phi E_{2}, E_{5}\right)}{g\left(\phi T, E_{4}\right)}>0, \text { if } r \geq 5
\end{gathered}
$$

where $g\left(\phi T, E_{3}\right)=0, g\left(\phi T, E_{2}\right) \neq 0$ and $g\left(\phi T, E_{4}\right) \neq 0$ are constants, $\frac{c+3}{4}-\frac{\delta_{1}}{\delta_{2}}+\frac{3(c-1)}{4}\left[g\left(\phi T, E_{2}\right)\right]^{2}-\lambda^{2}>0$ and $\lambda>0$ are constants.

Proof. If we take $\alpha=1, \beta=0, f_{1}=\frac{c+3}{4}, f_{2}=f_{3}=\frac{c-1}{4}$ in Corollary 3.4, we obtain the desired results.

Remark 4.1. $k_{4}$ does not need to be constant. So, there exists interpolating sesqui-harmonic curves which are not helices in a Sasakian space form with $\operatorname{dim} N \geq 5$.

Let $\left(N^{2 n+1}, \phi, \xi, \eta, g\right)$ be a Kenmotsu space form. Thus we have $\alpha=0, \beta=1, f_{1}=\frac{c-3}{4}, f_{2}=f_{3}=\frac{c+1}{4}$. From [1] and [2] we obtain $f_{2}=\frac{c+1}{4}=0$, that is $c=-1$.

By the use of Theorem 3.2, we obtain the following theorem:

Theorem 4.3. Let $\left(N^{2 n+1}, \phi, \xi, \eta, g\right)$ be a cosymplectic space form with $c=-1$ and $\gamma: I \subset \mathbb{R} \longrightarrow N^{2 n+1}(-1)$ be a Legendre curve of osculating order $r$.

(1) $\gamma$ is interpolating sesqui-harmonic if and only if it is a circle with $k_{1}=\sqrt{-1-\frac{\delta_{1}}{\delta_{2}}}$ where $\frac{\delta_{1}}{\delta_{2}}<-1$ or;

(2) $\gamma$ is interpolating sesqui-harmonic if and only if it is a helix with $k_{1}^{2}+k_{2}^{2}=-1-\frac{\delta_{1}}{\delta_{2}}$ where $\frac{\delta_{1}}{\delta_{2}}<-1$.

Let $\left(N^{2 n+1}, \phi, \xi, \eta, g\right)$ be a cosymplectic space form. Hence $\alpha=\beta=0, f_{1}=f_{2}=f_{3}=\frac{c}{4}$. From the equation (3.19), we obtain $k_{1} \eta\left(E_{2}\right)=0$, that is $\eta\left(E_{2}\right)=0$, with $k_{1}>0$.

For the Case VI, using Theorem 3.7, we obtain the following theorem:

Theorem 4.4. Let $\left(N^{2 n+1}, \phi, \xi, \eta, g\right)$ be a cosymplectic space form with $c \neq 0, \phi T \perp E_{2}, \xi \perp E_{2}$ and $\gamma: I \subset \mathbb{R} \longrightarrow$ $N^{2 n+1}(c)$ a Legendre curve of osculating order $r$.

(1) $\gamma$ is interpolating sesqui-harmonic with $\frac{\delta_{1}}{\delta_{2}} \neq 0$ if and only if it is a circle with $k_{1}=\sqrt{\frac{c}{4}-\frac{\delta_{1}}{\delta_{2}}}$ where $\frac{c}{4}>\frac{\delta_{1}}{\delta_{2}}$ or;

(2) $\gamma$ is interpolating sesqui-harmonic with $\frac{\delta_{1}}{\delta_{2}} \neq 0$ if and only if it is a helix with $k_{1}^{2}+k_{2}^{2}=\frac{c}{4}-\frac{\delta_{1}}{\delta_{2}}$ where $\frac{c}{4}>\frac{\delta_{1}}{\delta_{2}}$.

For the Case VIII, from Corollary 3.4, we obtain the following theorem:

Theorem 4.5. Let $\left(N^{2 n+1}, \phi, \xi, \eta, g\right)$ be a cosymplectic space form with $c \neq 0, \phi T \in\left\{E_{2}, E_{3}, E_{4}\right\}, g\left(\phi T, E_{2}\right) \neq 0, \xi \perp E_{2}$ and $\gamma: I \subset \mathbb{R} \longrightarrow N^{2 n+1}$ a Legendre curve of osculating order $r$.

or

(1) $\gamma$ is interpolating sesqui-harmonic with $\frac{\delta_{1}}{\delta_{2}} \neq 0$ if and only if it is a circle with $k_{1}=\sqrt{c-\frac{\delta_{1}}{\delta_{2}}}$ where $c>\frac{\delta_{1}}{\delta_{2}}, \phi T \| E_{2}$;

(2) $\gamma$ is interpolating sesqui-harmonic with $\frac{\delta_{1}}{\delta_{2}} \neq 0$ if and only if it is a Frenet curve of order $r \geq 4$ with

$$
\begin{gathered}
k_{1}=\lambda>0, \\
k_{2}=\sqrt{\frac{c}{4}-\frac{\delta_{1}}{\delta_{2}}+\frac{3 c}{4}\left[g\left(\phi T, E_{2}\right)\right]^{2}-\lambda^{2}}>0,
\end{gathered}
$$




$$
\begin{gathered}
k_{3}=\frac{-\frac{3 c}{4} g\left(\phi T, E_{2}\right) g\left(\phi T, E_{4}\right)}{\sqrt{\frac{c}{4}-\frac{\delta_{1}}{\delta_{2}}+\frac{3 c}{4}\left[g\left(\phi T, E_{2}\right)\right]^{2}-\lambda^{2}}}>0, \\
k_{4}=\frac{\lambda g\left(\phi E_{2}, E_{5}\right)}{g\left(\phi T, E_{4}\right)}>0, \text { if } r \geq 5
\end{gathered}
$$

where $g\left(\phi T, E_{3}\right)=0, g\left(\phi T, E_{2}\right) \neq 0$ and $g\left(\phi T, E_{4}\right) \neq 0$ are constants, $\frac{c}{4}-\frac{\delta_{1}}{\delta_{2}}+\frac{3 c}{4}\left[g\left(\phi T, E_{2}\right)\right]^{2}-\lambda^{2}>0$ and $\lambda>0$ are constants.

\section{References}

[1] Alegre, P., Blair, D.E., Carriazo, A.: Generalized Sasakian space forms. Israel J. of Math. 141, 157-183 (2004).

[2] Alegre, P., Carriazo, A.: Structures on generalized Sasakian space forms. Differ. Geom. Appl. 26 (6), 656-666 (2008).

[3] Blair, D.E.: The theory of quasi-Sasakian structures. J. Differ. Geom. 1, 331-345 (1967).

[4] Blair, D.E.: Riemannian Geometry of Contact and Symplectic Manifolds. Boston, Birkhauser (2002).

[5] Branding, V.: Some analytic results on interpolating sesqui-harmonic maps. Preprint arxiv:1907.04167 (2019).

[6] Branding, V.: On interpolating sesqui-harmonic maps between Riemannian manifolds. J. Geom. Anal. 30, 248-273 (2020).

[7] Eells, J. Jr., Sampson, J. H.: Harmonic mappings of Riemannian manifolds. Amer. J. Math. 86, 109-160 (1964).

[8] Jiang, GY.: 2-Harmonic maps and their first and second variational formulas. Chinese Ann. Math. Ser. A. 7, 389-402 (1986).

[9] Kenmotsu, K.: A class of almost contact Riemannian manifolds. Tôhoku Math. J. 24, 93-103 (1972).

[10] Karaca, F., Özgür, C., De, U. C.: On Interpolating Sesqui-Harmonic Legendre Curves in Sasakian Space Forms. International Journal of Geometric Methods in Modern Physics. 17(1), 2050005 (2020).

[11] Laugwitz, D.: Differential and Riemannian geometry. Academic Press. New York (1965).

[12] Loubeau, L., Montaldo, S.: Biminimal immersions. Proc. Edinb. Math. Soc. 51, 421-437 (2008).

[13] Ludden, G.D.: Submanifolds of cosymplectic manifolds. J. Dif. Geo. 4, 237-244 (1970).

[14] Marrero, J.C.: The local structure of trans-Sasakian manifolds. Ann. Mat. Pura Appl. 162, 77-86 (1992).

[15] Oubiña, J.A.: New classes of almost contact metric structures. Publ. Math. Debrecen. 32 (3-4), 187-193 (1985).

[16] Özgür, C., Güvenç, Ş.: On some classes of biharmonic Legendre curves in generalized Sasakian space forms. Collect. Math. 65 (2), 203-218 (2014).

\section{Affiliations}

FATMA KARACA

AdDress: Beykent University, Dept. of Mathematics, 34550, Istanbul-Turkey.

E-MAIL: fatmagurlerr@gmail.com

ORCID ID: 0000-0002-0382-8028 